Canadian Journal of Psychology

1979, 33, 201-205.

\title{
Octave Generalization and the Consolidation of Melodic Information
}

\author{
DIANA DEUTSCH \\ University of California, San Diego
}

\begin{abstract}
Subjects listened to a standard six-tone melody, followed by a comparison melody. The comparison melody was always transposed up four semitones from the standard, and this transposition was either exact or it was inexact while maintaining contour. Subjects judged whether or not the comparison melody formed an exact transposition of the standard. It was found that repetition of the standard melody, before presentation of the comparison, resulted in a significant improvement in performance compared with a condition featuring only a single presentation of the standard. A significant improvement also resulted when the repeated melody was displaced intact an octave higher or an octave lower. However, when the standard melody was repeated such that its components were displaced alternately to the higher and lower octaves, performance was significantly worse than when the standard melody was not repeated at all. Thus, in this paradigm, octave equivalence effects for single tones did not operate in the consolidation of melodic information.
\end{abstract}

It is clear from a variety of evidence that a strong perceptual similarity exists between tones that are separated by octaves (Deutsch, 1973). Several investigators have therefore suggested that pitch be treated as a bidimensional attribute: the first dimension represents overall pitch level, or 'tone height,' and the second defines the position of a tone within the octave, or 'tone chroma' (Meyer, 1904, 1914; Révész, 1913; Ruckmick, 1929; Bachem, 1948). Contemporary music theorists make an analogous distinction by referring to 'pitch' and 'pitch class' (Forte, 1973).

Given all the evidence for octave generalization in music, one might be tempted to assume that it holds true for all types of musical processing. However, Deutsch (1969) proposed rather that the abstraction of pitch information takes place along two separate and parallel channels. Along one of these channels there is convergence of information from neural units underlying tones separated by octaves; this gives rise to octave generalization. The har- monic equivalence of chords whose components are placed in different octaves would then be mediated by higher-order convergence of units along this channel. Along the second channel there is no such octave convergence, but instead abstraction of information required for transposition takes place; that is, abstraction of the ratios between tonal frequencies.

This two-channel model therefore predicts that where judgments of melodic relationships are concerned, octave generalization effects should not operate (except under special conditions, as described in Deutsch, 1978). One prediction which arises from this model is that we should find it very difficult to recognize well-known melodies when their component tones are placed randomly in different octaves. This was substantiated in an experiment by Deutsch (1972) and also more recently by Dowling and Hollombe (1977).

The present study was undertaken to determine whether octave generalization effects for single tones occur in the short-term 
consolidation of melodic information. (For other studies investigating the short-term retention of melodic information, see Cuddy and Cohen, 1976, and Dewar, Cuddy, and Mewhort, 1977). If listeners are presented with a standard melody, followed by a comparison melody, we should expect repetition of the standard before presentation of the comparison to result in enhanced performance, compared with a condition where the standard melody is presented only once. We can then ask whether such an enhancement also occurs when the component tones of the repeated melody are displaced to different octaves.

In this experiment, to ensure that the repetition effects studied involved relations between tones rather than absolute pitch values, the comparison melody was always transposed up four semitones from the standard. Further, the paradigm chosen was such that the contour of the comparison melody (as defined by the sequence of directions of pitch change) was always identical with that of the standard melody, regardless of whether or not the comparison melody was an exact transposition of the standard. This eliminated the potential use of contour as a basis for discrimination (Werner, 1925; White, 1960; Dowling and Fujitani, 1971).

\section{METHOD}

\section{Conditions}

In all conditions, subjects were presented with a standard six-tone sequence and later with a comparison sequence. The comparison sequence was always transposed up four semitones from the standard. On half of the trials, this transposition was exact, so that the set of melodic intervals and their orders were preserved. On the other half, two of the tones in the transposed sequence were permuted. The permuted tones were always a semitone apart in pitch, and no tones were permuted that were either at the beginning or at the end of the sequence, or that were adjacent to each other. Thus in the permuted sequences, four out of the five melodic intervals were changed in size by a semitone; however, the contour of the melody remained unaltered regardless of whether or not the comparison sequence was an exact transposition of the standard. Subjects judged whether or not the standard and comparison sequences formed exact transpositions. They indicated their judgments by writing ' $\mathrm{S}$ ' (same) or ' $\mathrm{D}$ ' (different) on paper.

There were four conditions in the experiment. In Condition 1, the standard sequence was presented once, followed by the comparison sequence. In Condition 2, the standard sequence was repeated six times (making seven presentations in all) before presentation of the comparison sequence. In Condition 3, the standard sequence was again repeated six times, but now on half of these repetitions the entire sequence was displaced an octave higher, and on the other half it was displaced on octave lower. On half of the trials, the order of placement for the repeated sequences was H L H L H L, and on the other half it was L H L H L H. In Condition 4 the standard sequence was again repeated six times, but now on each repetition the individual tones in the sequence were displaced alternatelyinto the higher and lower octaves. On half of the trials the order of placement for the individual tones was $\mathrm{H} \mathrm{L} \mathrm{H} \mathrm{L} \mathrm{H} \mathrm{L}$, and on the other half it was L H L H L H.

There were 12 trials in each condition. On six of these the comparison sequence formed an exact transposition of the standard, and on the other six the comparison sequence contained permuted tones. The trials were presented in random order, with no separation by condition.

\section{Temporal Parameters}

All sequences consisted of $500 \mathrm{msec}$ tones, with no gaps between tones. All sequences within trials were separated by six-second pauses. A warning click always occurred three seconds before the onset of the comparison sequence. Trials were presented in groups of four, with ten-second pauses between trials within a group, and two-minute pauses between groups. The testing period lasted approximately one hour.

\section{Frequencies}

All tones were taken from an equal-tempered scale (International Pitch, $\mathrm{A}=435$ ) and ranged over three octaves from $\mathrm{C}=129 \mathrm{~Hz}$ to $\mathrm{B}=977 \mathrm{~Hz}$. Except as specified below, the tones forming each standard sequence were chosen at random without replacement from the twelve semitones within the octave between middle $\mathrm{C}(259 \mathrm{~Hz})$ and the $\mathrm{B}$ above $(488 \mathrm{~Hz})$. The tones forming each comparison sequence ranged from E $(326 \mathrm{~Hz})$ to $\mathrm{D \#}(615 \mathrm{~Hz})$. On trials where the comparison sequence formed an exact transposition of the standard, all tones in the comparison sequence were exactly four semitones above those of the 
standard. On trials where the comparison sequence contained permuted tones, these tones were always a semitone apart in pitch. The pitches of the permuted tones were strictly counterbalanced across conditions, so that each condition contained comparison sequences where the following tone pairs were permuted (here presented in order of occurrence): $\mathrm{E}=326 \mathrm{~Hz}$ and $\mathrm{F}=345 \mathrm{~Hz} ; \mathrm{G}=388 \mathrm{~Hz}$ and $\mathrm{F} \#=366 \mathrm{~Hz} ; \mathrm{G} \#=411 \mathrm{~Hz}$ and $\mathrm{A}=435 \mathrm{~Hz} ; \mathrm{B}=488 \mathrm{~Hz}$ and $\mathrm{A} \#=461 \mathrm{~Hz} ; \mathrm{C}=517 \mathrm{~Hz}$ and $\mathrm{C} \#=548 \mathrm{~Hz} ; \mathrm{D} \#=615$ $\mathrm{Hz}$ and $\mathrm{D}=581 \mathrm{~Hz}$. Thus on half of these sequences the first of the permuted tones was lower in pitch than the second, and on the other half the second was lower than the first. Further, the serial positions of the permuted tones were strictly counterbalanced across conditions, so that for each condition, on two of the trials these tones were in serial positions 2 and 4; on two they were in positions 3 and 5 , and on two, they were in positions 2 and 5 . The pitches of the tones in the repeated sequences were as specified by the experimental conditions.

\section{Subjects}

Twenty-five undergraduates at the University of California at San Diego served as subjects for the experiment, and were paid for their services. They were selected on the basis of obtaining a score of at least $67 \%$ correct on a tape containing twelve sequences, designed as in Condition 1. Prior to the experiment the subjects were presented with several examples of each condition to familiarize them with the procedure. It was explained that in all repetitions, each note of the melody would be either identical with its corresponding note in the standard melody, or separated from this note by exactly an octave. Subjects were encouraged to take advantage of all repetitions in preparing to make their comparison judgments.

\section{Apparatus}

Tones were generated at equal amplitude by a Wavetek oscillator (model number 155) controlled by a PDP-8 computer, and were recorded on high fidelity tape. The tape was played to subjects on a high quality Revox tape recorder, the output of which was passed through a Fisher Stereo Master Control Amplifier, with controls adjusted so that the tones were equal in loudness. The output was played to subjects through loudspeakers. Subjects listened in groups of five or fewer in a quiet room.

\section{RESULTS}

The percentage errors in the different conditions of the experiment are plotted on Figure 1. It can be seen that exact repetition of the standard sequence resulted in a substantial improvement in comparison performance. This effect was found to be highly significant (Conditions 1 vs $2 ; p<.01$; two-tailed on a Wilcoxon test). An improvement also resulted when the standard sequence was repeated such that on each repetition it was displaced in its entirety to either the higher or the lower octave. This effect was also found to be highly significant (Conditions 1 vs. $3, p<.01$, twotailed on a Wilcoxon test) though the degree of improvement here was significantly less than with straight repetition (Conditions 2 vs. $3, p<.02$, two-tailed on a Wilcoxon test). However, when the standard sequence was

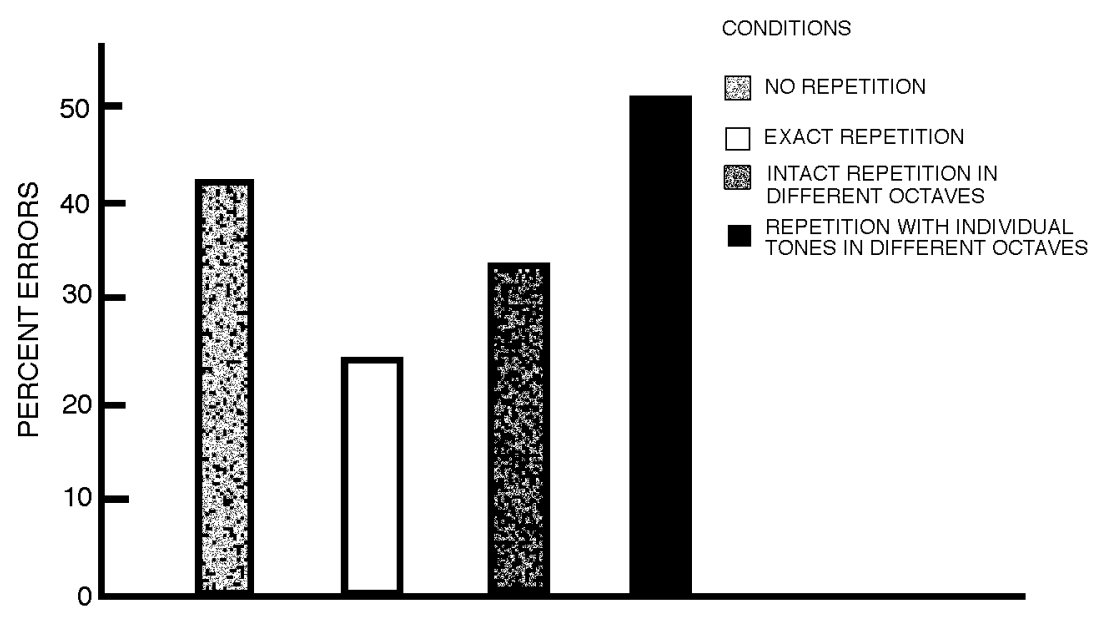

Figure 1. Percentage errors in the different conditions of the experiment. 
repeated such that its component tones alternated between the higher and lower octaves, performance was significantly poorer than when the standard sequence was not repeated at all (Conditions 1 vs. $4, p<.05$, two-tailed on a Wilcoxon test).

\section{DISCUSSION}

The results of the present experiment are consistent with the two-channel model proposed by Deutsch (1969) for the abstraction of pitch information. According to this model, melodic intervals are abstracted in two stages. In the first stage, neural units responding to tones of specific pitch project in pairs onto second-order units, each of which responds to the presentation of a given succession of tones. In the second stage, second-order units responding to successive tone pairs which stand in the same ratio project onto the same third-order units. These thirdorder units therefore respond to successive tone pairs which stand in a given ratio, regardless of the absolute pitches of their components. It is further assumed that consolidation of melodic information is based on a strengthening of signals emitted by units underlying memory for information abstracted in this fashion. Now in Condition 4 the component tones of the melody were displaced to different octaves, resulting in a different set of ratios formed by the successive tone pairs. We should not, therefore, expect memory consolidation to occur in this condition. Indeed, we might expect the different intervals in the interpolated melodies to disrupt memory for the intervals in the standard melody, in the same way as recognition memory for a tone can be disrupted by the interpolation of other tones (Deutsch, 1970). Performance in Condition 4 would in this case be poorer than in Condition 3, where the comparison melody followed the standard with no interpolated melodies. This finding was, in fact, obtained.
An additional finding to arise from the present experiment is that the improvement in comparison performance which results from repeating the standard sequence is significantly greater when these repetitions are exact than when the repeated sequences are displaced in their entirety to different octaves (Conditions 2 vs. 3). This effect cannot be due directly to a consolidation of the absolute pitch information in the exact repetition condition, since the set of pitches forming the comparison sequence always differed from those forming the standard sequence. However, we could hypothesize that consolidation of melodic relationships takes place more effectively when the same lower-order units underlying absolute pitch are repetitively stimulated. In the case of visual shape, it has also been hypothesized that such information might be more effectively retained at the locus of primary excitation, though the experimental evidence on this question is ambiguous (Wallach \& Austin, 1954; Hochberg, 1974).

\section{REFERENCES}

BACHEM, A. Note on Neu's review of the literature on absolute pitch. Psycho. Bull., 1948, 45, 161-162

CUDDY, L. L., \& COHEN, A. J. Recognition of transposed melodic sequences. Quart. J. exp. Psychol., 1976, 28, 255-270

DEUTSCH, D. Music recognition. Psychol. Rev., 1969, 76, 300-307

DEUTSCH, D. Tones and numbers: specificity of interference in short-term memory. Science, 1970, 168, 1604-1605

DEUTSCH, D. Octave generalization and tune recognition. Percept. Psychophys., 1972, 11, 411-412

DEUTSCH, D. Octave generalization of specific interference effects in memory for tonal pitch. Percept. Psychophys., 1973, 13, 271-272

DEUTSCH, D. Octave generalization and melody identification. Percept. Psychophys., 1978, 23, 91-92

DEWAR, K. M., CUDDY, L. L., \& MEWHORT, D. J. K., Recognition memory for single tones with and without context. J. exp. Psychol.: Human Learn. Mem., 1977, 3, 60-67

DOWLING, W. J., \& FUJITANI, D. S. Contour, interval and pitch recognition memory for melodies. J. Acoust. Soc. Am., 1971, 49, 524-531 
DOWLING, W. J., \& HOLLOMBE, A. W. The perception of melodies distorted by splitting into several octaves. Effects of increasing continuity and melodic contour. Percept. Psychophys., 1977, 21, 60-64

FORTE, A. The structure of atonal music. New Haven: Yale University Press, 1973

HOCHBERG, J. Organization and the gestalt tradition. In E. C. CARTERETTE \& M. P. FRIEDMAN (Eds.), Handbook of perception, Vol. 1, New York: Academic Press, 1974 pp. 180-211

MEYER, M. On the attributes of the sensations. Psychol. Rev., 1904, 11, 83-103

MEYER, M. Review of G. Revesz, 'Zur Grundleguncy der Tonpsychologie.' Psychol. Bull., 1914, 11, 349352

RÉVÉSZ, G. Zur Grundleguncy der Tonpsychologie. Leipzig: Feit, 1913

RUCKMICK, C.A. A new classification of tonal qualities. Psychol. Rev., 1929, 36, 172-180

WALLACH, H., \& AUSTIN, P. Recognition and the localization of visual traces. Am. J. Psychol., 1954, 57, 338-340

WERNER, H. Uber Mickromelodik und Mickroharmonik. Zeitshrift fur Psychologie, 1925, 28, 74-89

WHITE, B. Recognition of distorted melodies. Am. J. Psychol., 1960, 73, 100-107 
6 DEUTSCH 
